\title{
A study on image-processing based identification of aspect ratio of coarse aggregate
}

\author{
Tianhua $\mathrm{Li}^{1}$, Wenying Bai ${ }^{1}$, Kejia Zhang ${ }^{1}$ and Zichao Pan $^{2 *}$ \\ ${ }^{1}$ Xinjiang urban construction and test Co., Ltd., 830000 Urumqi, China \\ ${ }^{2}$ Department of Bridge Engineering, Tongji University, 200092, Shanghai, China
}

\begin{abstract}
The mesoscopic simulation of behaviours of cementitious materials under different conditions has become a hot topic in academic research, as it provides more details to the mechanism study and structural design. To conduct a mesoscopic simulation, the meso-scale model of cementitious materials must be built. To ensure the precision of the aggregate shape in the simulated meso-scale model, key shape parameters of real aggregates should be identified. In this paper, an image-processing based method is proposed to detect the aspect ratio of a polygonal aggregate. The procedure and used algorithms are demonstrated in detail. As an application, totally about 1000 coarse aggregates from the Xinjiang, China are selected to identify the aspect ratio. It is found that the aspect ratio of coarse aggregates is a random variable following the Generalized Extreme Value (GEV) distribution. The published data by using the X-ray technique is also adopted as a comparison, and the results are almost the same as each other, which indicates that the aggregate source does not have an obvious effect on the probabilistic characteristics of the aspect ratio.
\end{abstract}

\section{Introduction}

The numerical simulation of cementitious materials at meso-scale has become a hot topic in these days. Compared with a classical macroscopic simulation, the analysis at meso-scale can provide more details about the behaviours of cementitious materials in different conditions. For example, in the field of fracture mechanics, the propagation of cracks in concrete was simulated by a mesoscopic approach [1-3]. In the durability performance, both of chloride diffusion and concrete carbonation have been studied at meso-scale in many publications [4-8]. With the rapid development of powerful computers and advanced computational techniques, the numerical simulation of cementitious materials at meso-scale may provide useful tools in academic research for a deeper mechanism study and structural design to consider the statistical aspect of the material heterogeneity.

The first step of an analysis at meso-scale is to build a meso-scale model of cementitious materials. Generally, it is recognized that concrete at meso-scale can be considered as a heterogeneous material which consists of the aggregate, cement paste and interfacial transition zone (ITZ). The cement paste is simplified as a homogeneous material. Thus, the effect of the microstructure of concrete, e.g., pore network, is not explicitly considered at mesoscale. The ITZ is modelled as a shell with an identical thickness around the aggregate. Experimental studies found that the thickness of ITZ is dependent on the size of the cement particle instead of the aggregate. The thickness of ITZ is reported in the range of $10 \sim 50 \mu \mathrm{m}[9,10]$. Based on the above analysis, the essential point of building a meso-scale model of concrete is how to simulate the aggregate. The shape of a real aggregate is usually so complicated that a simplification is needed for an efficient simulation. Different parameters have been proposed in publications to represent the characteristics of aggregate shape, e.g., roundness, sphericity, angularity, convexity ratio, surface texture [11]. The surface texture is usually not considered in a meso-scale model of concrete. Thus, the aggregate shape can be simplified by basic geometries such as circle [12,13], ellipse [6], polygon [4], sphere [8] and ellipsoid [14]. It should be mentioned that, as solid particles, aggregates in the meso-scale model of concrete are not allowed to be overlapped with one another. Thus, when building the meso-scale model, an algorithm for the separation check is needed to ensure that each aggregate is separated from any other aggregate. For a complex aggregate shape, the algorithm for the separation check may be difficult to implement. That is why the circle is still used in recent publication to simulate the aggregate shape although it is over-simplified $[8,15]$. Theoretical and numerical simulation researches already found that the chloride diffusivity of concrete can be affected by the aspect ratio of aggregates $[6,16]$, which cannot be considered by circular aggregates. Thus, as a balance between the precision and efficiency, it is reasonable to simplify the aggregate shape in a 2D problem as an ellipse for pebbles with smooth surfaces and polygon for crushed-stones with rough surfaces. It is noticeable that although some three-dimensional aggregate models are already available, they usually require too much computational work to be accepted in a mesoscopic

\footnotetext{
* Corresponding author: z.pan@tongji.edu.cn
} 
analysis. Thus, a 2D analysis is still popular in recent publications due to efficiency consideration.

To obtain the characteristics of the real aggregate shape, the X-ray technique can be used to calibrate the three principal dimensions of an aggregate particle, i.e., the longest dimension, intermediate dimension and shortest dimension [11]. With these principal dimensions, the flatness and elongation of an aggregate particle can then be defined. This method can obtain the threedimensional information of the aggregate shape, but it requires that an X-ray scan is available. As an alternative approach, this paper proposed an image-processing based method to acquire the aspect ratio of the aggregate which is simulated as a polygon.

\section{Models and methods}

\subsection{Equipment system}

The test equipment system is schematic shown in Fig. 1. The image is taken by a scanner and transferred to a computer, where the image is processed to obtain the outline of an aggregate particle. To ensure the quality of the image and further analysis, the test is conducted in a darkroom where the only light is provided by the scanner to minimize the influence of shadow on the results of the image processing.

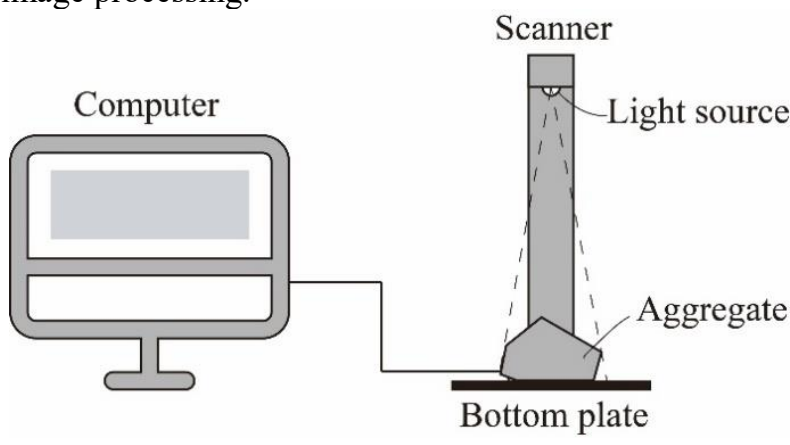

Fig. 1. A schematic of image-processing test system.

\subsection{Aggregate source}

The coarse aggregates investigated in this paper are coming from the Xinjiang Uygur Autonomous region in China. Totally about 1000 aggregate particles are selected, and the sizes of these aggregates have covered the gradation commonly used in reinforced concrete structures. Some images of the selected aggregates are shown in Fig. 2.

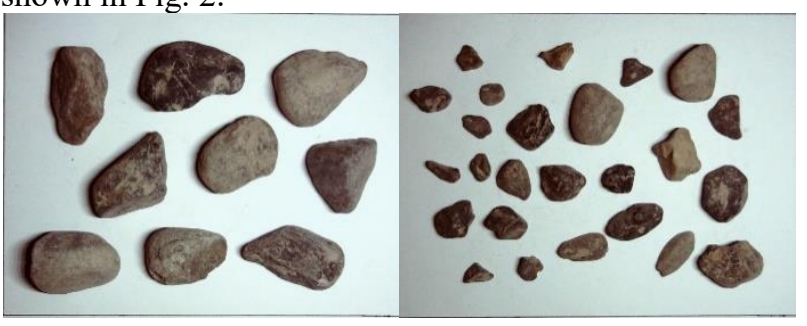

Fig. 2. Images of selected aggregates.

\subsection{Test method}

\subsubsection{Definition of aspect ratio}

The aggregate is simplified as a polygon with $N$ vertices in this paper. For each side of the polygon, a bounding rectangle can be found, as shown in Fig. 3. If the width and height of the bounding rectangle are denoted as $W_{i}$ and, $H_{i}\left(W_{i}>H_{i}\right)$ respectively, the aspect ratio of the polygonal aggregate can be defined as of the bounding rectangle with the smallest area, i.e.,

$$
\beta=W_{i} / H_{i} \text { with } \min A_{i}(i=1, \ldots, N)
$$
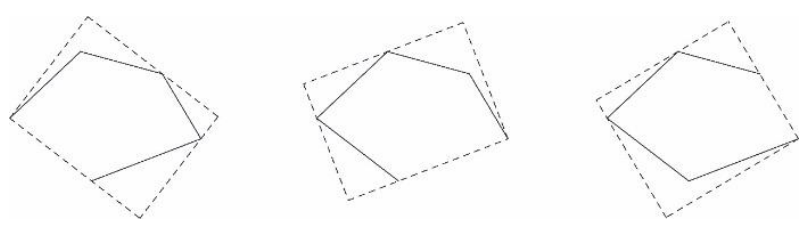

Fig. 3. A schematic of bounding rectangle of polygon.

\subsubsection{Image acquisition}

To minimize the influence of the shadow on the result of image processing, the test is conducted in the darkroom where the scanner is the only light source. In an ideal condition, it is expected that the connection between the point light source and aggregate is perpendicular to the bottom plate, to which the aggregate is projected. But since the scanner is a kind of scattered light source, the shadow in the images still exist and is unavoidable. To deal with this problem. an "optimal image area" is selected from the bottom plate. This area is determined mainly based on two considerations: (1) in terms of efficiency, the area cannot be too small, and it should be guaranteed that one image can hold sufficient aggregates to improve the efficiency of the image acquisition; (2) in terms of accuracy, the area cannot be too large, otherwise the area of shadow in the image will be unacceptable. With the above two considerations, the optimal image area used in the test is chosen as $182 \times 139 \mathrm{~mm}$. The aggregates are only allowed to be placed in this region.

In the computer, the bitmap image is stored in the unit of pixel. Thus, to obtain the size of the real aggregate in the further image processing, the relationship between the image size (in pixels) and the real size (in $\mathrm{mm}$ ) should be established. Thus, the scanner is configurated to fix the resolution for each image. The optimal image area in the image has the pixel size of $1084 \times 836$. Thus, the pixel density can be calculated as ppi $=5.98 \mathrm{pixel} / \mathrm{mm}$. In this way, the real size of the aggregate can be obtained by simply counting the number of pixels covered by the aggregate in the image.

\subsubsection{Image processing}

The raw image obtained from the test system is shown in Fig. 4(a). Based on Eq. (1), to calculate the aspect ratio of each aggregate in the image, the bounding rectangle with the smallest size should be found. Thus, a binarization operation is firstly conducted to transform the colored 
image into a black-and-white mode. Then, the morphological closing is performed on the binary image to ensure that the outline of the aggregate is closed, which can result in the image in Fig. 4(b). Secondly, the algorithm "Connected Component Labelling (CCL)" [17] is adopted to separate aggregates in the image into different images, as shown in Fig. 4(c). Thirdly, the bounding rectangle with the smallest area can be found, as shown in Fig. 4(c). Based on the width and height of the bounding rectangle, the aspect ratio of the polygon can be calculated by Eq. (1).

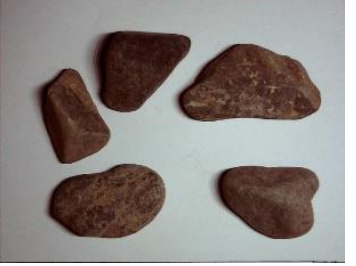

(a) raw image

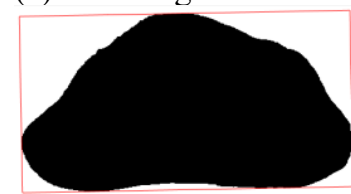

(c) aggregate separation

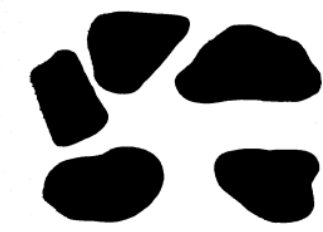

(b) binary image

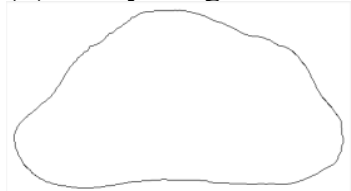

(d) edge detection
Fig. 4. Procedure of image processing.

After the aspect ratio of the polygon is determined, to further obtain the real size of the aggregate, the Sobel edge detection algorithm is used to identify the outline of the polygon, as shown in Fig. 4(d). Then, the real size of the aggregate can be approximately calculated as

$$
d=0.5 \times\left(\sqrt{\frac{4 N_{\mathrm{A}}}{\pi}}+\frac{N_{\mathrm{P}}}{\pi}\right) / \mathrm{ppi}
$$

where $N_{\mathrm{A}}$ and $N_{\mathrm{P}}$ are the total number of pixels on the outline and inside the aggregate, respectively.

\section{Results and discussion}

\subsection{Probabilistic characteristics}

Based on the above method, the real sizes of 1026 aggregate particles are tested, and the histogram plot of the 1026 data points are shown in Fig. 5. The result indicates that the sizes of the tested aggregates are mostly in the range of $10 \sim 40 \mathrm{~mm}$.

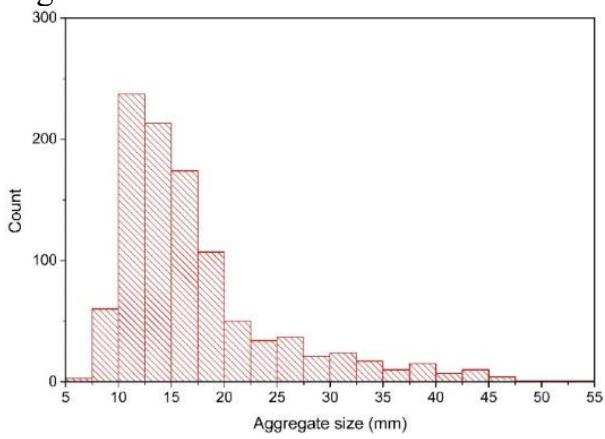

Fig. 5. Histogram plot of aggregate size calculated by Eq. (2).

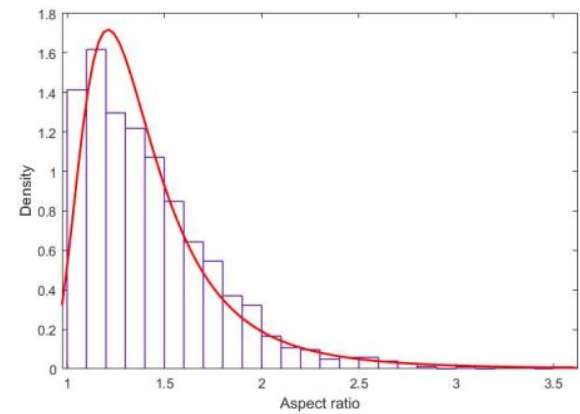

Fig. 6. Histogram plot of aspect ratio of aggregate.

Similar to the aggregate size, the histogram of the aspect ratio of aggregates is plotted in Fig. 6. As can be seen, the aspect ratios of the aggregates are mostly in the range of 1.0 2.0. If the aspect ratio is considered as random variable, the Generalized Extreme Value (GEV) distribution can be used to fit the data, and the result is:

$$
\beta \sim \operatorname{GEV}(\mu=1.25, \sigma=0.22, \xi=0.22)
$$

Based on the theory of GEV, the mean value can be calculated as:

$$
\mathrm{AVG}=\mu+\frac{\sigma[\Gamma(1-\xi)-1]}{\xi}=1.4375
$$

where $\Gamma(\mathrm{x})$ is the Gamma function, while the median value can be calculated as:

$$
\operatorname{MED}=\mu+\sigma \frac{(\ln 2)^{-\xi}-1}{\xi}=1.3340
$$

The above results can provide objective information to build the meso-scale model cementitious materials.

\subsection{Comparison with published data}

By using the X-ray technique, Erdogan [11] calibrated the principal dimensions of aggregates from different districts in US. The results, compared with the results obtained in this paper, are plotted in Fig. 7. It can be found that the statistical distribution of the aspect ratio in [11] is similar to that in this paper. Thus, the GEV distribution is also tried to conduct the data fitting, and the result is:

$$
\beta \sim \operatorname{GEV}(\mu=1.26, \sigma=0.18, \xi=0.18)
$$

Based on the theory of GEV, the mean value can be calculated as:

$$
\mathrm{AVG}=\mu+\frac{\sigma[\Gamma(1-\xi)-1]}{\xi}=1.4375
$$

where $\Gamma(\mathrm{x})$ is the Gamma function, while the median value can be calculated as:

$$
\operatorname{MED}=\mu+\sigma \frac{(\ln 2)^{-\xi}-1}{\xi}=1.3340
$$

The above results can provide objective information to build the meso-scale model cementitious materials. 


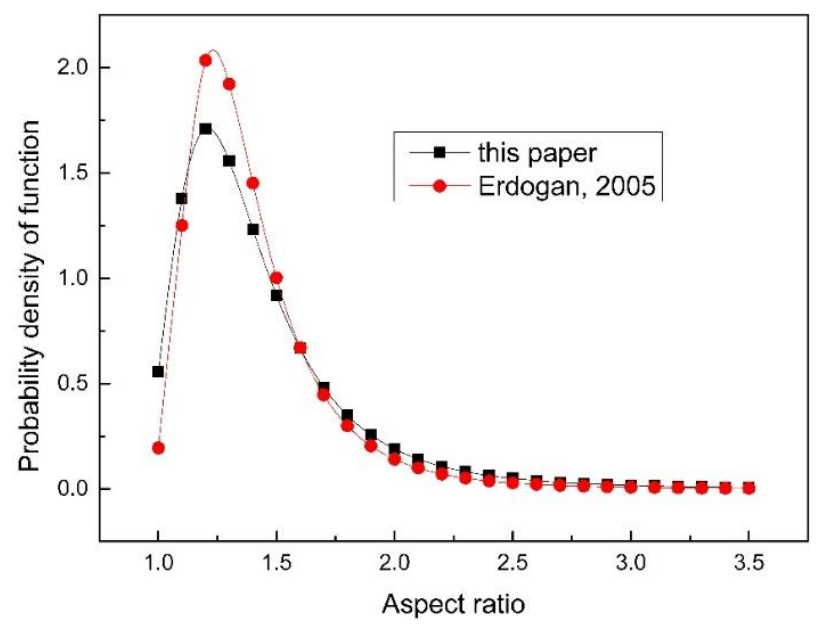

Fig. 7. Comparison between results of aspect ratio of aggregate obtained in this paper and [11].

\section{Conclusions}

This paper proposed an image-processing based method to obtain the aspect ratio of a coarse aggregate. This method has the advantages of low requirement of equipment and high efficiency. By this method, the aspect ratios of about 1000 aggregates from the Xinjiang Uygur Autonomous region in China are obtained and statistically analyzed. Based on the results and discussion, the following conclusions can be drawn:

(1) The aspect ratio of an aggregate particle can be seen as a random variable following GEV distribution.

(2) The mean and median values of the aspect ratio are 1.4375 and 1.3340 , respectively.

(3) The aspect ratios of about $66.57 \%$ and $93.57 \%$ of aggregates are in the range of $1.0 \sim 1.5$ and 1.0 2.0, respectively.

(4) By comparing with the other published data by the $\mathrm{X}$-ray technique, it is found that the probabilistic characteristics of the aspect ratio are almost the same, and the aggregate source does not show an obvious effect on the aspect ratio.

The authors would like to thank the National Natural Science Foundation of China (No. 51608377) and the Fundamental Research Funds for the Central Universities of China for the financial support.

\section{References}

[1] G. Lilliu, J.G.M.V. Mier, 3D lattice type fracture model for concrete, Eng. Fract. Mech. 70(7-8) (2003) 927-941.

[2] A. Chen, Z. Pan, R. Ma, Mesoscopic simulation of steel rebar corrosion process in concrete and its damage to concrete cover, Struct. Infrastruct. Eng. 13(4) (2017) 478-493.

[3] B. Šavija, M. Luković, J. Pacheco, E. Schlangen, Cracking of the concrete cover due to reinforcement corrosion: A two-dimensional lattice model study, Constr. Build. Mater. 44 (2013) 626-638.

[4] Z. Pan, A. Chen, X. Ruan, Spatial variability of chloride and its influence on thickness of concrete cover:
A two-dimensional mesoscopic numerical research, Engineering Structures 95 (2015) 154-169.

[5] Z. Pan, X. Ruan, A. Chen, A 2-D numerical research on spatial variability of concrete carbonation depth at meso-scale, Comput. Concr. 15(2) (2015) 231-257.

[6] Z. Pan, X. Ruan, A. Chen, Chloride diffusivity of concrete: probabilistic characteristics at meso-scale, Comput. Concr. 13(2) (2014) 187-207.

[7] B. Šavija, M. Luković, E. Schlangen, Lattice modeling of rapid chloride migration in concrete, Cem. Concr. Res. 61-62 (2014) 49-63.

[8] B. Šavija, J. Pacheco, E. Schlangen, Lattice modeling of chloride diffusion in sound and cracked concrete, Cem. Concr. Compos. 42 (2013) 30-40.

[9] J.P. Ollivier, J.C. Maso, B. Bourdette, Interfacial Transition Zone in Concrete, Advanced Cement Based Materials 2(1) (1995) 30-38.

[10] K.L. Scrivener, K.M. Nemati, The percolation of pore space in the cement paste/aggregate interfacial zone of concrete, Cem. Concr. Res. 26(1) (1996) 35-40.

[11] S.T. Erdogan, Determination of aggregate shape properties using X-ray tomographic methods and the effect of shape on concrete rheology, The University of Texas at Austin, Austin, US, 2005.

[12] Z.P. Bazant, M.R. Tabbara, M.T. Kazemi, G. Pijaudiercabot, Random Particle Model for Fracture of Aggregate or Fiber Composites, J. Eng. Mech. 116(8) (1990) 1686-1705.

[13] X. Ruan, Z. Pan, Mesoscopic simulation method of concrete carbonation process, Struct. Infrastruct. Eng. 8(2) (2012) 99-110.

[14] S. Hafner, S. Eckardt, T. Luther, C. Konke, Mesoscale modeling of concrete: Geometry and numerics, Comput. Struct. 84(7) (2006) 450-461.

[15] J. Han, W. Liu, S. Wang, D. Du, F. Xu, W. Li, G. De Schutter, Effects of crack and ITZ and aggregate on carbonation penetration based on 3D micro X-ray CT microstructure evolution, Constr. Build. Mater. 128 (2016) 256-271

[16] J. Zheng, X. Zhou, Y. Wu, X. Jin, A numerical method for the chloride diffusivity in concrete with aggregate shape effect, Constr. Build. Mater. 31(6) (2012) 151-156.

[17] H. Samet, M. Tamminen, Efficient component labeling of images of arbitrary dimension represented by linear bintrees, IEEE Transactions on Pattern Analysis \& Machine Intelligence 10(4) (1988) 579-586. 